\title{
Encyclopedia of Metagenomics
}



Sarah K. Highlander

Francisco Rodriguez-Valera

Bryan A. White

Editors

\section{Encyclopedia of Metagenomics}

Environmental Metagenomics

With 163 Figures and 46 Tables

Springer Reference 


\section{Editors}

Sarah K. Highlander

Genomic Medicine

J. Craig Venter Institute

La Jolla, CA, USA

Francisco Rodriguez-Valera

Universidad Miguel Hernandez

Campus San Juan

San Juan, Alicante, Spain
Bryan A. White

The Institute for Genomic Biology

Department of Animal Sciences \&

Pathobiology

Division of Nutritional Sciences

University of Illinois

Urbana, IL, USA

ISBN 978-1-4899-7474-7

ISBN 978-1-4899-7475-4 (eBook)

ISBN 978-1-4899-7476-1 (print and electronic bundle)

DOI 10.1007/978-1-4899-7475-4

Springer New York Heidelberg Dordrecht London

Library of Congress Control Number: 2014954612

\section{(C) Springer Science+Business Media New York 2015}

This work is subject to copyright. All rights are reserved by the Publisher, whether the whole or part of the material is concerned, specifically the rights of translation, reprinting, reuse of illustrations, recitation, broadcasting, reproduction on microfilms or in any other physical way, and transmission or information storage and retrieval, electronic adaptation, computer software, or by similar or dissimilar methodology now known or hereafter developed. Exempted from this legal reservation are brief excerpts in connection with reviews or scholarly analysis or material supplied specifically for the purpose of being entered and executed on a computer system, for exclusive use by the purchaser of the work. Duplication of this publication or parts thereof is permitted only under the provisions of the Copyright Law of the Publisher's location, in its current version, and permission for use must always be obtained from Springer. Permissions for use may be obtained through RightsLink at the Copyright Clearance Center. Violations are liable to prosecution under the respective Copyright Law.

The use of general descriptive names, registered names, trademarks, service marks, etc. in this publication does not imply, even in the absence of a specific statement, that such names are exempt from the relevant protective laws and regulations and therefore free for general use.

While the advice and information in this book are believed to be true and accurate at the date of publication, neither the authors nor the editors nor the publisher can accept any legal responsibility for any errors or omissions that may be made. The publisher makes no warranty, express or implied, with respect to the material contained herein.

Printed on acid-free paper

Springer is part of Springer Science+Business Media (www.springer.com) 


\section{Preface}

Volume 2 encompasses metagenomics of humans, animals, oceans, soils and continental waters. While the composition of the metagenomes of these environments differs greatly, the theme of discovery of important new uncultivated members is common to all. High-throughput 16S rRNA sequencing of communities has been used to define bacterial and archaeal membership. Metagenomics is providing deeper information about community structures, potential metabolic function and the interplay between members within particular environments. In humans and animals, this information has provided clues to health and disease. In soils, oceans and waters, stratified, diverse communities have been described, many of which come from extreme environments. Although the volume is organized alphabetically, the following are brief synopses of individual topic areas:

Human Metagenomics. The human microbiome is the composition of microbes (bacteria, archaea, fungi, protists and viruses) that live in and on the human body. These microbes are crucial to host immune development, nutrition and protection against pathogens. Reports of human microbiome studies began to increase exponentially beginning in the mid-2000s, when high-throughput sequencing technologies were applied to characterize distinct communities on the skin and in the oral, respiratory, genitourinary and gastrointestinal tracts. Disruption of these communities was soon shown to be associated with both chronic and acute diseases including some forms of cancer. The field has yielded new methodologies, such as tools for highthroughput 16S rRNA gene sequencing and analysis, single-cell capture of uncultured bacteria for sequencing and new algorithms for metagenomic assembly, clustering and taxonomic classification.

The chapters address major colonized human body sites and important individual phyla, families or species of bacteria that are members of the human microbiome. Over a dozen entries discuss the microbiome and its association with disease states. In some cases, direct microbial causal links are shown, while in others, disease-associated changes are described. Reviews of the microbiome of infants, pregnancy and mode of delivery and the intestinal microbiome of the aged are included.

Animal Metagenomics. The gastrointestinal tract (GIT) microbiome of animals is becoming increasingly recognized as a critical factor in overall health, development and productivity. Early gut colonization is critically important to the morphological and immunological development of the GIT, and as the GIT microbiome matures, it exhibits significant diversity, 
an important indicator of ecosystem health. The development of next-generation sequencing technologies and meta-omic approaches has enabled the exploration of these microbiomes at unprecedented depths. This in turn has enabled holistic system-based approaches that have provided fresh insights into the properties that define collective colonizing microbial communities as they relate to health and disease. These entries cover the current state of knowledge of both domesticated and wild animals and how the GIT microbiome impacts health, survival, fecundity, population fitness and, ultimately, evolution of the host.

Metagenomics of the Ocean. Marine microbiology has a particular handicap for using classical techniques based on culture. Due to low concentrations of nutrients that prevail in most oceanic waters, marine microbes tend to be oligotrophs that grow very poorly in standard laboratory media. The concept of the Plate Count Anomaly derives from marine microbiology. It is, therefore, not surprising that the first and perhaps more revealing metagenomic studies have been carried out in the ocean. Seawater is particularly easy to process for biomass collection, and by using absolute filters of different sizes, eukaryotic and prokaryotic cells and viruses can be studied independently. Some of the major water bodies in the world have been studied with depth profiles, and some geographical and latitudinal gradients have been described. Still, the amount of unknowns in the marine microbial realm is enormous. Although great advances have been possible through metagenomics and (lately) single-cell genomics, many marine microbes are only known using 16S rRNA gene sequences that provided almost no information about their metabolism and lifestyle. Furthermore, we know now that within each taxonomic group, there is enormous diversity (the pan-genome) that can only be accessed by sequencing many strains or by extensively analyzing high-coverage metagenomes. Fortunately, the power of newgeneration sequencers will no doubt speed up the process. The enormous tracts of the oligotrophic oceans that cover our planet are fortunately relatively homogeneous. Here, the main changes are found in a depth profile. The understanding of the community structure and functioning of the largest habitat on Earth is now within the reach of marine microbiologists.

Metagenomics of Soils and Continental Waters. Soils are one of the most active and unknown microbial environments. The complexity and heterogeneity of its microbial communities make their study one of the great challenges of modern Microbiology. Metagenomics provides a very effective way to analyze such complex communities by pooling them together, and some initiatives to describe some model soils are now in advanced stages. The task of analyzing and understanding microbe-plant interactions and the roles of diverse sets of microbes present is of titanic dimensions, but the rewards at the level of more efficient agricultural technologies and ecological management of land are proportionate to the effort.

On the other hand, life in the continents depends on the input of water from the atmosphere that is mostly channelled into rivers and lakes. These aquatic environments are of critical importance for human well-being, providing drinking water and irrigation since Neolithic times. However, the field of freshwater microbiology has received little attention. Here again, the 
techniques and advances of marine metagenomics can be used to an advantage, providing a better understanding of major microbial players and their roles in keeping natural freshwater bodies in healthy ecological condition. This knowledge can also be of use to determine potential detrimental activities to human health, such as the production of toxins by cyanobacterial blooms. It is clear that the nascent field of freshwater metagenomics will develop as an essential tool for ecology and sanitation.

CA, USA

Sarah K. Highlander

Alicante, Spain

Francisco Rodriguez-Valera

IL, USA

Bryan A. White

September 2014 



\section{About the Editor}

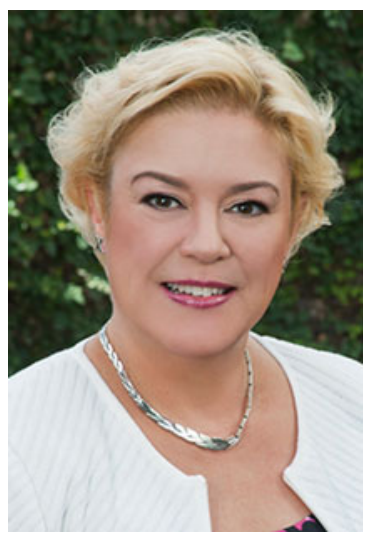

Sarah K. Highlander is a Professor in the Genomic Medicine Group at the J. Craig Venter Institute (JCVI) in La Jolla, California. Before joining JCVI, she was an Associate Professor in the Department of Molecular Virology and Microbiology and the Human Genome Sequencing Center (HGSC) at Baylor College of Medicine (BCM) in Houston, Texas. She has broad expertise in medical and veterinary microbiology, bacterial genetics, and microbial genomics resulting from her experiences in academia, the pharmaceutical industry and as a founder of an animal health startup. Dr. Highlander's current interests are focused on applied microbiome research, comparative microbial genomics, and computational metagenomics methods for pathogen discovery in diarrhea of unknown etiology. She is also involved in additional oral, skin, and gastrointestinal microbiome and metagenomics projects at JCVI.

At BCM, Dr. Highlander was a principal investigator for the Human Microbiome Project (HMP) and held leadership positions in the Consortium. She was responsible for oversight of reference genome sequencing at the HGSC and her laboratory created "mock communities" of DNA and cells that are used as benchmarking standards for microbiome studies. She also has a long record of experience studying the pathogenesis of a polymicrobial pneumonia in cattle, called bovine respiratory disease, or shipping fever. The primary bacterial agent in this disease is the gram-negative bacterium, Mannheimia haemolytica, which is the same family as the human respiratory pathogen, Haemophilus influenzae. Her group performed extensive characterization of the $M$. haemolytica leukotoxin and its expression and developed numerous genetic tools for manipulation and fluorescent tagging of the organism. She holds patents for subunit and live-attenuated vaccines to prevent shipping fever. Dr. Highlander was the lead investigator on several important microbial genome sequencing and annotation projects, including that of Mannheimia haemolytica, the community acquired methicillin resistant Staphylococcus aureus clone USA300, the oral pathogen Fusobacterium nucleatum subsp. polymorphum, and the radiation resistant Bacillus pumilus strain SAFR-032. 
Dr. Highlander is a member of the Editorial Boards of Infection and Immunity and Microbial Ecology and served on the Editorial Board of PLoS $O N E$ from 2010 to 2012. She reviews manuscripts for dozens of microbiology and genomics journals and has served on numerous review panels for the NIH, NSF, and USDA. At Baylor College of Medicine, she played key roles in education and administration within the Graduate School of Biomedical Sciences. Dr. Highlander earned her B.S. in Cellular Biology from the University of Michigan, Ann Arbor, and M.S. and Ph.D. degrees in Medical Microbiology from the Sackler Institute of Biomedical Sciences at the New York University School of Medicine.

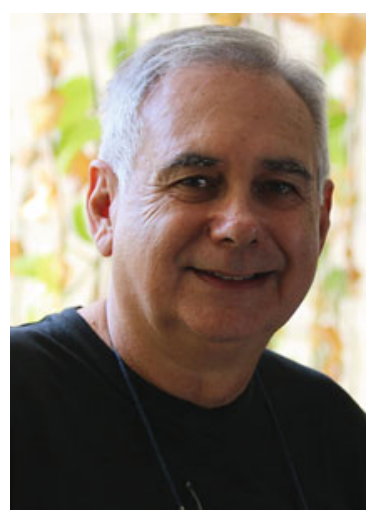

Francisco Rodriguez-Valera's career in Microbiology has been one of continued excellence and exploration of new fields. He was a pioneer in the study of halophilic microbes since the early 1980s leading to what is now the accepted pattern of classification of halophilic Archaea. He was leader in the isolation and study of the most relevant microbes in these environments (Haloferax, Haloarcula, Haloquadratum, Salinibacter, and, just last year, Spiribacter). Very early, he applied molecular approaches first and later genomics and metagenomics to the study of aquatic ecosystems including hypersaline (solar salterns), marine (deep chlorophyll maximum), and fresh waters (the Amazon River). He spearheaded the use of new-generation sequencing in metagenomics by using high-throughput sequencing for both direct and fosmid metagenome sequencing. By fosmid sequencing, he and his group have managed to sequence thousands of complete uncultivated marine viruses producing the single largest increase in the number of complete marine bacteriophage genomes. He also used genomics and metagenomics to dissect the population genomics of marine and halophilic populations. His studies of the population genomics of Haloquadratum, the marine copiotroph Alteromonas and in-depth analysis of metagenomes and metaviromes has led to a proposal of how the populations of marine prokaryotes are structured at the level of genomic diversity. The model of constant diversity claims that aquatic microbes, even when belonging to a single taxonomic unit such as a species, form concurrent metaclonal populations (made up of many different clones). These clones are different in their ecological properties (e.g., they can use different substrates) and in their phage receptors, e.g., the O-chain of the lipopolysaccharide. Both sets of genomic features are linked together at the ecological timescale (weeks to years) and provide the population with stable and efficient combined attributes. The viral component is essential to equalize the different clonal subpopulations by a "kill the winner" feedback control.

As head of the Evolutionary Genomics Group, Rodriguez-Valera is leading several initiatives to develop an active community of metagenomicists in Europe and other areas of the world, particularly in Latin America. He and his 
team organize courses and workshops to familiarize newcomers to the field with the methodological challenges of metagenomics.

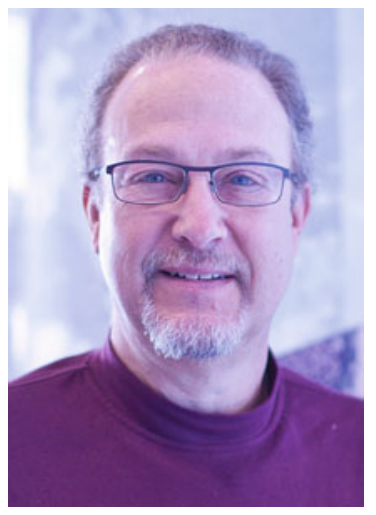

Bryan A. White is currently a Professor of Animal Sciences in the Institute for Genomic Biology and Director, Mayo Clinic/University of Illinois Strategic Alliance for TechnologyBased Healthcare. He received his Bachelor of Science at Virginia Wesleyan College and his $\mathrm{Ph} . \mathrm{D}$. in Microbiology from the Medical College of Virginia and was an NIH Postdoctoral Fellow in the Department of Oral Biology at the University of Michigan. My major research interests are in using microbial physiology and genetics, microbial genomics and metagenomics, and microbial ecology to understand host-microbe interactions in vertebrates. These interests are in production species such as cattle, swine, and poultry and in models of disease for humans. With respect to production species, we are interested in models that address nutrient utilization, primarily the rumen fiber-adherent microbiome as a model for improving forage utilization. We are also interested in understanding the roles of beneficial and pathogenic organisms and antibiotic use in domestic production animals as well as approaches that will enable the detection of diseases in livestock and provide critical genetic contexts for understanding food safety. With respect to humans and nonhuman primates, we are using the framework of the Human Microbiome Project from the NIH Road Map to address issues in health and well-being. One interest is how bacterial communities colonizing the gastrointestinal and reproductive tracts of primates impact health, survival, fecundity, population fitness, and ultimately evolution of the host. Our other major interest is in characterizing the microbiome in the human gastrointestinal and reproductive tracts and the relationship between the microbiome and urogenital infections, preterm deliveries and women's health, the development of systemic autoimmune diseases, and understanding the role of dietary components in cancer etiology, prevention, and cancer health disparities. We apply high-throughput genomic technologies to identify microbes and make gene predictions that can lead to personalized medical diagnostics and genomic information that physicians could use as predictors of risk and positively impact clinical outcomes. 



\section{Contributors}

Kjersti M. Aagaard Department of Obstetrics and Gynecology, Baylor College of Medicine, Houston, TX, USA

Olukemi O. Abolude Institute for Genome Sciences, School of Medicine, University of Maryland, Baltimore, MD, USA

Laura S. Achenbaum Social Sciences Research Institute, Rice University, Houston, TX, USA

J. Félix Aguirre-Garrido Departamento de Microbiología del Suelo y Sistemas Simbióticos, Estación Experimental del Zaidín, Consejo Superior de Investigaciones Científicas (CSIC), Granada, Spain

Departamento de Sistemas Biológicos, Universidad Autónoma Metropolitana \& Xochimilco, Mexico

Jackie Aislabie Landcare Research, University of Waikato, Hamilton, New Zealand

Nadim J. Ajami Department of Molecular Virology and Microbiology, Alkek Center for Metagenomics and Microbiome Research, Houston, TX, USA

Ramasamy Anbuchezhian Marine Biotechnology Laboratory, State Key Laboratory of Microbial Metabolism, School of Life Sciences and Biotechnology, Shanghai Jiao Tong University, Shanghai, China

Amber Anders Department of Obstetrics and Gynecology, Baylor College of Medicine, Houston, TX, USA

Fernando Dini Andreote Department of Soil Science, University of Sao Paulo, Sao Paulo, Brazil

Angelina Academy of Scientific and Innovative Research, New Delhi, India Department of Food Microbiology, CSIR-Central Food Technological Research Institute, Mysore, Karnataka, India

Josefa Anton Department of Physiology, Genetics and Microbiology, University of Alicante, Alicante, Spain

Merlin Ariefdjohan Charles C. Gates Center for Regenerative Medicine and Stem Cell Biology, University of Colorado Denver, Aurora, CO, USA 
Virender Kumar Batish Molecular Biology Unit, Dairy Microbiology Division, National Dairy Research Institute, Karnal, Haryana, India

Alison N. Beloshapka Department of Animal Sciences, University of Illinois at Urbana-Champaign, Urbana, IL, USA

Clara Belzer Laboratory of Microbiology, Wageningen University, Wageningen, The Netherlands

Gabriele Berg Institute of Environmental Biotechnology, Graz University of Technology, Graz, Austria

Renaud Berlemont Department of Earth System Science and Department of Ecology and Evolutionary Biology, University of California, Irvine, Irvine, CA, USA

Miroslav Blumenberg Department of Dermatology, New York University, School of Medicine, New York, NY, USA

James G. Bockheim Soil Science, University of Wisconsin, Madison, WI, USA

Annemarie Boleij Department of Medicine, Division of Infectious Diseases, Johns Hopkins University School of Medicine, Baltimore, MD, USA

Silja Brady Department of Genomic and Applied Microbiology, Institute of Microbiology and Genetics, Georg-August-Universität Göttingen, Göttingen, Germany

Anastasia Bragina Institute of Environmental Biotechnology, Graz University of Technology, Graz, Austria

Steven E. Brenner Department of Plant and Microbial Biology, University of California, Berkeley, CA, USA

Georgia Olympia Brikis Technical University of Munich, Munich, Germany

Eoin Brodie Ecology Department, Lawrence Berkeley National Laboratory, Berkeley, CA, USA

Todd R. Callaway Food and Feed Safety Research Unit, USDA, Southern Plains Agricultural Research Center, Agricultural Research Service, College Station, TX, USA

Isaac Cann Departments of Animal Science and Microbiology, University of Illinois, Urbana-Champaign, IL, USA

Franck Carbonero Department of Food Science, University of Arkansas, Fayetteville, AR, USA

Ana Maria Cardenas University of Pennsylvania Health System, University of Pennsylvania, Philadelphia, PA, USA

Kimberly M. Carlson-Banning Department of Biochemistry and Molecular Biology, Baylor College of Medicine, Houston, TX, USA 
Emilio Ortega Casamayor Biodiversity and Biogeodynamics Group, Center for Advanced Studies of Blanes-Spanish Council for Research, CEAB-CSIC, Girona, Spain

Chun H. Chen Departments of Bioengineering, and Electrical and Computer Engineering, University of California, San Diego, CA, USA

Hsin-I Chiang Department of Animal Sciences, National Chung Hsing University, Taiwan

Departments of Bioengineering, and Electrical and Computer Engineering, University of California, San Diego, CA, USA

Marcus J. Claesson Department of Microbiology and Alimentary Pharmabiotic Centre, University College Cork, Cork, Ireland

Gerard Clarke Alimentary Pharmabiotic Centre, University College Cork, Cork, Ireland

Violetta La Cono Department of Marine Molecular Microbiology, Institute for Coastal Marine Environment IAMC-CNR, Messina, Italy

Jacob Cram Department of Biological Sciences, University of Southern California, Dana and David Dornsife College of Letters, Arts and Sciences, Los Angeles, CA, USA

Heather H. Creasy Institute for Genome Sciences, School of Medicine, University of Maryland, Baltimore, MD, USA

John Cryan Department of Anatomy and Neuroscience, University College Cork, Cork, Ireland

Zelin Cui Department of Laboratory Medicine, Shanghai First People's Hospital, Medical College, Shanghai Jiaotong University, Shanghai, China

Department of Medical Microbiology and Parasitology, Shanghai Jiao Tong University School of Medicine, Shanghai, China

Siobhán Cusack School of Microbiology and Alimentary Pharmabiotic Centre, University College Cork, Corcaigh, Ireland

Rolf Daniel Department of Genomic and Applied Microbiology, Institute of Microbiology and Genetics, Georg-August-Universität Göttingen, Göttingen, Germany

Ted Dinan Department of Psychiatry, University College Cork, Cork, Ireland

Elizabeth A. Dinsdale Biology Department, San Diego State University, San Diego, CA, USA

Alan D. W. Dobson Marine Biotechnology Centre, Environmental Research Institute, and School of Microbiology, University College Cork, Cork, Ireland 
Mitchel Doktycz BioSciences Division, Oak Ridge National Laboratory, Oak Ridge, TN, USA

Maria Dominguez-Bello Division of Translational Medicine, New York University School of Medicine, New York, NY, USA

Lisa Durso Agroecosystem Management Research Unit, US Department of Agriculture, University of Nebraska, Lincoln, NE, USA

Bas E. Dutilh Centre for Molecular and Biomolecular Informatics (CMBI), Radboud University Medical Centre, Nijmegen, The Netherlands

Tom S. Edrington Food and Feed Safety Research Unit, USDA, Southern Plains Agricultural Research Center, Agricultural Research Service, College Station, TX, USA

Annette S. Engel Department of Earth and Planetary Sciences, University of Tennessee, Knoxville, TX, USA

\section{European Cystic Fibrosis Society Lung Microbiome Working Group}

Paul Falkowski Departments of Geological Sciences and Marine and Coastal Sciences, Institute of Marine and Coastal Sciences, Rutgers University, New Brunswick, NJ, USA

Helisson Faoro Department of Biochemistry and Molecular Biology, Federal University of Parana, Curitiba, PR, Brazil

Natalie Fedorova-Abrams SAIC-Frederick, Frederick National Laboratory for Cancer Research, Frederick, MD, USA

Manuel Ferrer Institute of Catalysis, Spanish National Research Council, CSIC, Madrid, Spain

Kai Finster Department of Bioscience, Microbiology Section, Aarhus University, Aarhus, Denmark

Catherine M. Flaitz Oral and Maxillofacial Pathology, Department of Diagnostic and Biomedical Sciences, The University of Texas School of Dentistry at Houston, Houston, TX, USA

Larry J. Forney Institute for Bioinformatics and Computational Biology, Department of Biological Sciences, University of Idaho, Moscow, ID, USA

J. Dennis Fortenberry Adolescent Medicine, Indiana University School of Medicine, Indianapolis, IN, USA

Daniel Frank School of Medicine, Division of Infectious Diseases, University of Colorado, Denver, Denver, CO, USA

Jed A. Fuhrman Department of Biological Sciences, University of Southern California, Dana and David Dornsife College of Letters, Arts and Sciences, Los Angeles, CA, USA

David Galas Pacific Northwest Diabetes Research Institute, Seattle, WA, USA 
Radhika Ganu Department of Obstetrics and Gynecology, Baylor College of Medicine, Houston, TX, USA

H. Rex Gaskins Institute for Genomic Biology, University of Illinois at Urbana-Champaign, University of Illinois Cancer Center, Urbana, IL, USA

Rohit Ghai Departamento de Producción Vegetal y Microbiología, Evolutionary Genomics Group, Universidad Miguel Hernández, San Juan de Alicante, Alicante, Spain

Bibaswan Ghoshal Department of Agricultural, Food and Nutritional Science, University of Alberta, Edmonton, AB, Canada

Michelle G. Giglio Institute for Genome Sciences, School of Medicine, University of Maryland, Baltimore, MD, USA

Jack Gilbert Department of Ecology and Evolution, University of Chicago, Chicago, IL, USA

Laura Giuliano Department of Marine Molecular Microbiology, Institute for Coastal Marine Environment IAMC-CNR, Messina, Italy

Trine Glad Department of Arctic and Marine Biology, UiT The Arctic University of Norway, Tromsø, Norway

Filipa Godoy-Vitorino Department of Natural Sciences, Faculty of Sciences and Technology, Inter American University of Puerto Rico, Metropolitan Campus, San Juan, PR, USA

Peter N. Golyshin School of Biological Sciences, Bangor University, Bangor, Gwynedd, UK

José Eduardo González-Pastor Department of Molecular Evolution, Centro de Astrobiología (CSIC-INTA), Madrid, Spain

Sunita Grover Department of Dairy Microbiology, Molecular Biology Unit, National Dairy Research Institute, Karnal, Haryana, India

LeLuo Guan Department of Agricultural, Food and Nutritional Science, University of Alberta, Edmonton, AB, Canada

Masahira Hattori Center for Omics and Bioinformatics, Graduate School of Frontier Sciences, The University of Tokyo, Kashiwa, Chiba, Japan

Emma Hernandez-Sanabria Department of Agricultural, Food and Nutritional Science, University of Alberta, Edmonton, AB, Canada

Ian Hewson Department of Microbiology, Cornell University, Ithaca, NY, USA

Roxana Hickey Institute for Bioinformatics and Computational Biology, Department of Biological Sciences, University of Idaho, Moscow, ID, USA

Sarah K. Highlander Genomic Medicine, J. Craig Venter Institute, La Jolla, CA, USA 
Diane Hoffmann Francis King Carey School of Law, University of Maryland, Baltimore, MD, USA

Noah G. Hoffman Department of Laboratory Medicine, University of Washington, Seattle, WA, USA

Pei-Ying Hong Biological and Environmental Science and Engineering, King Abdullah University of Science and Technology (KAUST), Thuwal Saudi Arabia

Andrea Hsu Faculty of Dentistry, McGill University, Montreal, QC, Canada

Ting Huang Faculty of Dentistry, McGill University, Montreal, QC, Canada

Yvonne Huang Pulmonary and Critical Care Medicine, School of Medicine, University of California San Francisco, San Francisco, CA, USA

Zunxi Huang Engineering Research Center of Sustainable Development and Utilization of Biomass Energy, Ministry of Education, Kunming, China

School of Life Science, Yunnan Normal University, Kunming, China

Richard Hull Department of Molecular Virology and Microbiology, Baylor College of Medicine, Houston, TX, USA

Kristina G. Hulten Department of Pediatrics, Baylor College of Medicine, Houston, TX, USA

Diane S. Hutchinson Interdepartmental Program in Translational Biology and Molecular Medicine, Baylor College of Medicine, Houston, TX, USA

Richard Isaacson Department of Veterinary and Biomedical Sciences, University of Minnesota, St. Paul, MN, USA

Suzanne Ishaq Department of Animal Sciences, University of Vermont, Burlington, VT, USA

Stephen Jackson Marine Biotechnology Centre, Environmental Research Institute, and School of Microbiology, University College Cork, Cork, Ireland

Janet Jansson Earth Sciences Division, Lawrence Berkeley National Laboratory, Berkeley, CA, USA

Ian B. Jeffery Department of Microbiology and Alimentary Pharmabiotic Centre, University College Cork, Cork, Ireland

Benjamin I. Jelen Environmental Biophysics and Molecular Ecology Laboratory, Institute of Marine and Coastal Sciences, Rutgers University, New Brunswick, NJ, USA

Esther Jiménez Department of Nutrition, Food Science and Food Technology, Complutense University of Madrid, Madrid, Spain 
Brian V. Jones Center for Biomedical and Health Science Research, School of Pharmacy and Biomolecular Sciences, University of Brighton, Brighton, East Sussex, UK

Khady Ka Faculty of Dentistry, McGill University, Montreal, QC, Canada

Ulrich Gosewinkel Karlson Department of Environmental Science, Aarhus University, Roskilde, Denmark

Sarah W. Keenan Department of Earth and Planetary Sciences, University of Tennessee, Knoxville, TN, USA

Jonathan Kennedy Marine Biotechnology Centre, Environmental Angelina Khan Research Institute, and School of Microbiology, University College Cork, Cork, Ireland

Mahejibin Khan Academy of Scientific and Innovative Research, New Delhi, India

Department of Food Microbiology, CSIR-Central Food Technological Research Institute, Mysore, Karnataka, India

Roozbeh Khosravi Department of Molecular and Cell Biology, Division of Oral Biology, Boston University Henry M. Goldman School of Dental Medicine, Boston, MA, USA

Kyoung-Ho Kim Department of Microbiology, Pukyong National University, Busan, Republic of Korea

Min Seok Kim Meat Safety and Quality Research, USDA Agricultural Research Service, Clay Center, NE, USA

Athol Klieve School of Agriculture and Food Sciences, University of Queensland, Gatton, QLD, Australia

Rob Knight Department of Chemistry and Biochemistry, BioFrontiers Institute, Howard Hughe Medical Institute, University of Colorado at Boulder, Boulder, CO, USA

Zoya B. Kurago Diagnostic Sciences, College of Dental Medicine, Georgia Regents University, Augusta, GA, USA

Aroonwan Lam-ubol Faculty of Dentistry, Department of Oral Diagnosis, Srinakharinwirot University, Bangkok, Thailand

Federico M. Lauro School of Biotechnology and Biomolecular Sciences, The University of New South Wales, Sydney, NSW, Australia

Margie D. Lee Department of Population Health, The University of Georgia College of Veterinary Medicine, Athens, GA, USA

Natuschka Lee Lab Microbial Systems Ecology/Department of Microbiology, Technische Universität München, Freising/Munich, Germany

Seon-Woo Lee Department of Applied Biology, Dong-A University, College of Natural Resources and Life Science, Busan, Republic of Korea 
Uri Y. Levine Novozymes Biologicals, Inc., Salem, VA, USA

Cecil M. Lewis Department of Anthropology, University of Oklahoma, Norman, OK, USA

Huiying Li Department of Molecular and Medical Pharmacology, Crump Institute for Molecular Imaging, David Geffen School of Medicine, UCLA, Los Angeles, CA, USA

Weizhong Li Center for Research in Biological Systems (CRBS), University of California, La Jolla, CA, USA

Zhiyong Li Marine Biotechnology Laboratory, State Key Laboratory of Microbial Metabolism, School of Life Sciences and Biotechnology, Shanghai Jiao Tong University, Shanghai, China

An-dong Li Department of Civil Engineering, The University of Hong Kong, Hong Kong, China

Ellen Li Department of Medicine, Washington University School of Medicine, St. Louis, MO, USA

Shao Li Bioinformatics Division, TNLIST, MOE Key Laboratory of Bioinformatics, and Department of Automation, School of Life Sciences, Tsinghua University, Beijing, China

Wolfang Liebl Department of Microbiology, Technische Universität München, Freising/Munich, Germany

Manuel E. Lladser BioFrontiers Institute, Department of Applied Mathematics, University of Colorado at Boulder, Boulder, CO, USA

Yu-Hwa Lo Department of Electrical and Computer Engineering, University of California, San Diego, CA, USA

Manuel Fernández López Departamento de Microbiología del Suelo y Sistemas Simbióticos, Estación Experimental del Zaidín, Consejo Superior de Investigaciones Científicas (CSIC), Granada, Spain

Jun Ma Department of Obstetrics and Gynecology, Baylor College of Medicine, Houston, TX, USA

Roderick I. Mackie Department of Animal Science, University of Illinois, Urbana-Champaign, IL, USA

Ramana Madupu Genomic Medicine, J. Craig Venter Institute, Rockville, MD, USA

Alexander Mahnert Institute of Environmental Biotechnology, Graz University of Technology, Graz, Austria

Anup A. Mahurkar Institute for Genome Sciences, School of Medicine, University of Maryland, Baltimore, MD, USA

Rashmi H. Mallappa Molecular Biology Unit, Dairy Microbiology Division, National Dairy Research Institute, Karnal, Haryana, India 
Julian R. Marchesi School of Biosciences, Cardiff University, Cardiff, UK

Andrew P. Martin Department of Ecology and Evolutionary Biology, University of Colorado at Boulder, Boulder, CO, USA

Francisco Martínez-Abarca Departamento de Microbiología del Suelo y Sistemas Simbióticos, Estación Experimental del Zaidín, Consejo Superior de Investigaciones Científicas (CSIC), Granada, Spain

Manuel Martínez-García Department of Physiology, Genetics and Microbiology, University of Alicante, Alicante, Spain

Frederick A. Matsen Public Health Science Division, Fred Hutchinson Cancer Research Center, Seattle, WA, USA

Neil R. McEwan Institute of Biological, Environmental and Rural Sciences, Penglais Campus, Aberystwyth University, Aberystwyth, Wales, UK

Amy L. McGuire Center for Medical Ethics and Health Policy, Baylor College of Medicine, Houston, TX, USA

J. Chase McNeil Department of Pediatrics, Baylor College of Medicine, Houston, TX, USA

Mónica Medina Department of Biology, Pennsylvania State University, University Park, PA, USA

Jeremiah Minich Biology Department, San Diego State University, San Diego, CA, USA

John P. Morrissey Marine Biotechnology Centre, Environmental Research Institute, and School of Microbiology, University College Cork, Cork, Ireland

Paola Navarrete Instituto de Nutrición y Tecnología de los Alimentos, Universidad de Chile, Santiago, Chile

Taras Y. Nechitaylo Research Group Insect Symbiosis, Max Planck Institute for Chemical Ecology, Jena, Germany

Karen E. Nelson J. Craig Venter Institute, Rockville, MD, USA

Kristelle Q. Nguyen Faculty of Dentistry, McGill University, Montreal, QC, Canada

Belinda Nicolau Faculty of Dentistry, McGill University, Montreal, QC, Canada

Kaare M. Nielsen Department of Pharmacy, UiT The Arctic University of Norway, Tromsø, Norway

David J. Nisbet Food and Feed Safety Research Unit, USDA, Southern Plains Agricultural Research Center, Agricultural Research Service, College Station, TX, USA 
Beifang Niu Center for Research in Biological Systems (CRBS), University of California, La Jolla, CA, USA

Carlos Wolfgang Nossa Gene by Gene Ltd., Houston, TX, USA

Lisa Oberauner Austrian Centre of Industrial Biotechnology (ACIB $\mathrm{GmbH})$, Graz, Austria

Institute of Environmental Biotechnology, Graz University of Technology, Graz, Austria

Eibhlís M. O'Connor School of Microbiology and Alimentary Pharmabiotic Centre, University College Cork, Corcaigh, Ireland

Kieran C. O'Doherty Department of Psychology, University of Guelph, Guelph, ON, Canada

Fergal O'Gara BIOMERIT Research Centre and School of Microbiology, University College Cork, Cork, Ireland

Lesley A. Ogilvie Department of Vertebrate Genomics, Max Planck Institute for Molecular Genetics, Berlin, Germany

Aharon Oren Department of Plant and Environmental Sciences, Alexander Silberman Institute of Life Sciences, The Hebrew University of Jerusalem, Jerusalem, Israel

Paul W. O'Toole School of Microbiology and Alimentary Pharmabiotic Centre, University College Cork, Corcaigh, Ireland

Timothy Palzkill Department of Pharmacology, Baylor College of Medicine, Houston, TX, USA

Xiaoyan Pang State Key Laboratory of Microbial Metabolism, School of Life Sciences and Biotechnology, Shanghai Jiao Tong University, Shanghai, China

Soo-Je Park Department of Biology, Jeju National University, Jeju, Republic of Korea

W. J. van Mark Passel Laboratory of Systems and Synthetic Biology, Wageningen University, Wageningen, The Netherlands

Fábio Oliveira Pedrosa Department of Biochemistry and Molecular Biology, Federal University of Parana, Curitiba, PR, Brazil

Zhiheng Pei Departments of Pathology and Medicine, New York University School of Medicine, New York, NY, USA

Joseph F. Petrosino Department of Molecular Virology and Microbiology, Alkek Center for Metagenomics and Microbiome Research, Baylor College of Medicine, Houston, TX, USA

Stephen C. Pflugfelder Department of Ophthalmology, Baylor College of Medicine, Houston, TX, USA 
Mircea Podar Biosciences Division, Oak Ridge National Laboratory, Oak Ridge, TN, USA

Mihai Pop Center for Bioinformatics and Computational Biology, University of Maryland, College Park, MD, USA

Phillip B. Pope Department of Chemistry, Biotechnology and Food Science, Norwegian University of Life Sciences, Ås, Norway

Megan Porter Department of Biology, University of South Dakota, Vermillion, SD, USA

Pablo Power Department of Microbiology, CONICET, School of Pharmacy and Biochemistry, University of Buenos Aires, Buenos Aires, Argentina

Tulika Prakash School of Basic Sciences, Indian Institute of Technology (IIT) Mandi, Mandi, Himachal Pradesh, India

Lita M. Proctor Division of Genomic Sciences, National Institutes of Health (NIH), Bethesda, MD, USA

Egor B. Prokhortchouk Centre "Bioengineering" of the Russian Academy of Sciences, Moscow, Russia

Hugo C. Ramirez-Saad Departamento de Sistemas Biológicos, Universidad Autónoma Metropolitana \& Xochimilco, Mexico

Nikolai V. Ravin Centre of Bioengineering, Russian Academy of Sciences, Moscow, Russia

Sung-Keun Rhee Department of Microbiology, College of Natural Sciences, Chungbuk National University, Cheongju, Chungbuk, Republic of Korea

Rosamond Rhodes Medical Education, Mount Sinai School of Medicine, New York, NY, USA

Juan M. Rodríguez Department of Nutrition, Food Science and Food Technology, Complutense University of Madrid, Madrid, Spain

Jaime Romero Instituto de Nutrición y Tecnología de los Alimentos, Universidad de Chile, Santiago, Chile

Alejandro Salgado-Flores Department of Arctic and Marine Biology, UiT The Arctic University of Norway, Tromsø, Norway

Tina Santl-Temkiv Department of Physics and Astronomy, Department of Bioscience, Microbiology Section, Aarhus University, Aarhus, Denmark

Fernando Santos Department of Physiology, Genetics and Microbiology, University of Alicante, Alicante, Spain

R. Balfour Sartor Microbiology and Immunology, University of North Carolina, Charlotte, NC, USA

Richard R. Sharp Biomedical Ethics Program, Mayo Clinic, Rochester, MN, USA 
Jian Shen Department of Gynaecology and Obstetrics, Ruijin Hospital Affiliated to Shanghai Jiaotong University School of Medicine, Shanghai, China

Institute for Bioinformatics and Computational Biology, University of Idaho, Moscow, ID, USA

Irina N. Shilova Ocean Sciences Department, University of California Santa Cruz, Santa Cruz, CA, USA

Konstantin G. Skryabin Centre "Bioengineering" of the Russian Academy of Sciences, Moscow, Russia

Hauke Smidt Laboratory of Microbiology, Wageningen University, Wageningen, The Netherlands

Emanuel Maltempi de Souza Department of Biochemistry and Molecular Biology, Federal University of Parana, Curitiba, PR, Brazil

Paul Spicer Department of Anthropology, University of Oklahoma, Norman, OK, USA

Jennifer K. Spinler Department of Pathology and Immunology, Texas Children's Microbiome Center, Baylor College of Medicine, Houston, TX, USA

Thaddeus Stanton Food Safety and Enteric Pathogens Research Unit, USDA ARS, Ames, IA, USA

Christopher Stewart Applied Sciences, University of Northumbria, Newcastle upon Tyne, UK

Jan S. Suchodolski Gastrointestinal Laboratory, Department of Small Animal Clinical Sciences, College of Veterinary Medicine and Biomedical Sciences, Texas A\&M University, College Station, TX, USA

Fengzhu Sun Department of Biological Sciences, University of Southern California, Dana and David Dornsife College of Letters, Arts and Sciences, Los Angeles, CA, USA

Shinichi Sunagawa Structural and Computational Biology, European Molecular Biology Laboratory, Heidelberg, Germany

Monica A. Sundset Department of Arctic and Marine Biology, UiT The Arctic University of Norway, Tromsø, Norway

Kelly Swanson Department of Animal Sciences, University of Illinois at Urbana-Champaign, Urbana, IL, USA

Willem Takken Laboratory of Entomology, Wageningen University and Research Centre, Wageningen, The Netherlands

Kai Tang State Key Laboratory of Marine Environmental Science, Xiamen University, Xiamen, China 
Todd D. Taylor Laboratory for Integrated Bioinformatics, RIKEN Center for Integrative Medical Sciences, Yokohama, Kanagawa, Japan

Casey Theriot Division of Infectious Diseases, Department of Internal Medicine, University of Michigan Medical School, Ann Arbor, MI, USA

Anne W. Thompson Advanced Cytometry Group, BD Biosciences, Seattle, WA, USA

Luke Thompson BioFrontiers Institute, University of Colorado, Boulder, CO, USA

Igor Tiago Department of Life Sciences, University of Coimbra, Coimbra, Centro, Portugal

Harold Tjalsma Department of Laboratory Medicine, Nijmegen Institute for Infection, Inflammation and Immunity (N4i) and Research Institute for Oncology (RUCO), Radboud University Medical Centre, Nijmegen, The Netherlands

Shuta Tomida Department of Molecular and Medical Pharmacology, Crump Institute for Molecular Imaging, David Geffen School of Medicine, UCLA, Los Angeles, CA, USA

Nicolas Toro Departamento de Microbiología del Suelo y Sistemas Simbióticos, Estación Experimental del Zaidín, Consejo Superior de Investigaciones Científicas (CSIC), Granada, Spain

Simon D. Tran Faculty of Dentistry, McGill University, Montreal, QC, Canada

Xavier Triadó-Margarit Biodiversity and Biogeodynamics Group, Center for Advanced Studies of Blanes-Spanish Council for Research, CEAB-CSIC, Girona, Spain

Gena D. Tribble Department of Periodontics, University of Texas Health Science Center at Houston, School of Dentistry, Houston, TX, USA

Peter Um Department of Molecular Virology and Microbiology, Baylor College of Medicine, Houston, TX, USA

Niels O. Verhulst Laboratory of Entomology, Wageningen University and Research Centre, Wageningen, The Netherlands

James Versalovic Department of Pathology and Immunology, Baylor College of Medicine and Texas Children's Hospital, Houston, TX, USA

António Veríssimo Department of Life Sciences, University of Coimbra, Coimbra, Centro, Portugal

Erik C. von Rosenvinge Department of Medicine, University of Maryland School of Medicine, Baltimore, MD, USA

Sompong Vongpunsawad Department of Molecular Virology and Microbiology, Baylor College of Medicine, Houston, TX, USA 
Willem M. de Vos Laboratory of Microbiology, Wageningen University, Wageningen, The Netherlands

Department of Veterinary Biosciences, Department of Bacteriology and Immunology, Helsinki University, Helsinki, Finland

Kai Wang Institute for Systems Biology, Seattle, WA, USA

James E. Wells Meat Animal Research Center, USDA, Agricultural Research Service, Clay Center, NE, USA

Owen White Institute for Genome Sciences, School of Medicine, University of Maryland, Baltimore, MD, USA

Timothy J. Williams School of Biotechnology and Biomolecular Sciences, The University of New South Wales, Sydney, NSW, Australia

Paul Wilmes Luxembourg Centre for Systems Biomedicine, University of Luxembourg, Luxembourg

André-Denis G. Wright Department of Animal Sciences, University of Vermont, Burlington, VT, USA

Chenggang Wu Department of Microbiology and Molecular Genetics, University of Texas Health Science Center at Houston, Houston, TX, USA

Sitao Wu Center for Research in Biological Systems (CRBS), University of California, La Jolla, CA, USA

Bo Xu Engineering Research Center of Sustainable

Development and Utilization of Biomass Energy, Ministry of Education, Kunming, China School of Life Science, Yunnan Normal University, Kunming, China

Zhengsheng Xue State Key Laboratory of Microbial Metabolism, School of Life Sciences and Biotechnology, Shanghai Jiao Tong University, Shanghai, China

Patrick S. Yachimski Department of Medicine, Vanderbilt University School of Medicine, Nashville, TN, USA

Michail M. Yakimov Department of Marine Molecular Microbiology, Institute for Coastal Marine Environment IAMC-CNR, Messina, Italy

Liying Yang Department of Medicine, New York University School of Medicine, New York, NY, USA

Yuzhen Ye School of Informatics and Computing, Indiana University, Bloomington, IN, USA

Carl J. Yeoman Rumen/Gastrointestinal Microbiology, Montana State University, Bozeman, MT, USA

Suleyman Yildirim Department of Medical Microbiology, Medipol University School of Medicine, Istanbul, Turkey 
Shibu Yooseph Informatics, J. Craig Venter Institute, La Jolla, CA, USA

Bonnie P. Youmans Veterinary and Biomedical Sciences, University of Minnesota, Saint Paul, MN, USA

Vincent B. Young Department of Internal Medicine, Division of Infectious Diseases, Department of Microbiology and Immunology, University of Michigan Medical School, Ann Arbor, MI, USA

Ke Yu Environmental Biotechnology Laboratory, The University of Hong Kong, Pokfulam, Hong Kong

Zhongtang Yu Department of Animal Sciences, The Ohio State University, Columbus, OH, USA

Preeti Zanwar Sealy Center on Aging, University of Texas Medical Branch, Galveston, TX, USA

Lynn Zechiedrich Department of Molecular Virology and Microbiology, Verna and Department of Biochemistry and Molecular Biology, and Department of Pharmacology, Baylor College of Medicine, Houston, TX, USA

Jonathan P. Zehr Ocean Sciences Department, University of California Santa Cruz, Santa Cruz, CA, USA

Xuegong Zhang Bioinformatics Division, TNLIST, MOE Key Laboratory of Bioinformatics, and Department of Automation, School of Life Sciences, Tsinghua University, Beijing, China

Tong Zhang Environmental Biotechnology Laboratory, The University of Hong Kong, Pokfulam, Hong Kong

Liping Zhao State Key Laboratory of Microbial Metabolism, School of Life Sciences and Biotechnology, Shanghai Center for Systems Biomedicine, Shanghai Jiao Tong University, Shanghai, China

Mi Zhou Department of Agricultural, Food and Nutritional Science, University of Alberta, Edmonton, AB, Canada 